\title{
Serotonergic, Brain Volume and Attentional Correlates of Trait Anxiety in Primates
}

\author{
Yevheniia Mikheenko*,1,2, Yoshiro Shiba ${ }^{1,2,5}$, Stephen Sawiak ${ }^{2,4,5}$, Katrin Braesicke ${ }^{1,2,6}$, Gemma Cockcroft $^{2,3}$, \\ Hannah Clarke ${ }^{1,2}$ and Angela C Roberts ${ }^{1,2}$ \\ 'Department of Physiology, Development and Neuroscience, University of Cambridge, Cambridge, UK; ${ }^{2}$ Behavioural and Clinical Neuroscience \\ Institute, University of Cambridge, Cambridge, UK; ${ }^{3}$ Department of Psychology, University of Cambridge, Cambridge, UK; ${ }^{4}$ Wolfson Brain Imaging \\ Centre, University of Cambridge, Box 65 Addenbrooke's Hospital, Cambridge, UK
}

\begin{abstract}
Trait anxiety is a risk factor for the development and maintenance of affective disorders, and insights into the underlying brain mechanisms are vital for improving treatment and prevention strategies. Translational studies in non-human primates, where targeted neurochemical and genetic manipulations can be made, are critical in view of their close neuroanatomical similarity to humans in brain regions implicated in trait anxiety. Thus, we characterised the serotonergic and regional brain volume correlates of trait-like anxiety in the marmoset monkey. Low- and high-anxious animals were identified by behavioral responses to a human intruder (HI) that are known to be sensitive to anxiolytic drug treatment. Extracellular serotonin levels within the amygdala were measured with in vivo microdialysis, at baseline and in response to challenge with the selective serotonin reuptake inhibitor, citalopram. Regional brain volume was assessed by structural magnetic resonance imaging. Anxious individuals showed persistent, long-term fearful responses to both a $\mathrm{HI}$ and a model snake, alongside sustained attention (vigilance) to novel cues in a context associated with unpredictable threat. Neurally, high-anxious marmosets showed reduced amygdala serotonin levels, and smaller volumes in a closely connected prefrontal region, the dorsal anterior cingulate cortex. These findings highlight behavioral and neural similarities between trait-like anxiety in marmosets and humans, and set the stage for further investigation of the processes contributing to vulnerability and resilience to affective disorders.

Neuropsychopharmacology (20I5) 40, I395-|404; doi: I0. I038/npp.20 I4.324; published online I4 January 2015
\end{abstract}

\section{INTRODUCTION}

Trait-anxious individuals frequently experience anxiety across a range of different contexts, and display consistent biases in the interpretation of, and response to, potential threats (Grupe and Nitschke, 2013). Such biases contribute to the etiology and development of affective disorders (Sandi and Richter-Levin, 2009); thus, understanding the underlying neural mechanisms has the potential to improve both treatments and prevention of such disorders. Translational approaches linking human and animal research are key to this effort, and studies in non-human primates are of special importance, in view of their neuroanatomical similarity to humans in regions of the prefrontal cortex (PFC) involved in emotion regulation (Ongur and Price, 2000; Paxinos et al, 2011). Currently, the most prominent

*Correspondence: Dr Y Mikheenko, Department of Physiology, Development and Neuroscience, University of Cambridge, Downing Street, Cambridge CB2 3DY, UK, Tel: +44 1223 339015, Fax: +44 12233390 |4, E-mail: ypm20@cam.ac.uk

${ }^{5}$ The authors contributed equally to this work.

${ }^{6}$ Current address: Fraunhofer Institute of Optronics, System Technologies and Image Exploitation (IOSB), Gutleuthausstr. I, 76275 Ettlingen, Germany.

Received 4 September 20।4; revised I 8 November 20।4; accepted 29 November 20।4; accepted article preview online II December 2014 models of anxiety and fear in non-human primates focus on responses to human intruders (HIs) or innate responses to predators (Barros and Tomaz, 2002b; Fox and Kalin, 2014). However, exactly how these correspond to behavioral markers of anxiety studied in humans and rodents, such as response biases to more abstract conditioned cues or contexts associated with threat (Davis et al, 2010), remains unknown. We recently demonstrated that heightened anxiety in response to innate threat (snake), in the marmoset, is associated with undifferentiated cardiovascular and behavioral responses to threat and safety during aversive discriminative conditioning (Shiba et al, 2014). We now extend this model of trait-like anxiety, not only to examine individual differences in responses to different anxiety-provoking situations, but importantly to probe their underlying cardiovascular, neural and neurochemical basis. Thus, we compared the responses of high- and lowanxious animals to three threatening situations: exposure to a HI, a model snake and a chamber associated with the delivery of unpredictable aversive noise. During the latter, we also assessed behavioral (attentional) and heart rate (HR) responses to neutral cues presented in the threatassociated context, given that responses to unpredictable threat and ambiguous cues are often heightened in traitanxious humans (Grupe and Nitschke, 2013) and anxious patients, eg, (Grillon et al, 2009). 
At the neural level, trait anxiety is associated with changes in the amygdala (Grupe and Nitschke, 2013; Kim et al, 2011; Oler et al, 2010), a region prominent in the control of anxious responses to diffuse/prolonged cues or contexts (Davis et al, 2010). More specifically, modulation of the amygdala by the neurotransmitter serotonin is linked to both anxiety-related traits in healthy humans (Cools et al, 2005), and treatment outcomes in patients (Faria et al, 2014). Higher anxiety is associated with increased serotonin transporter (5-HTT) binding (Oler et al, 2009) and lower post mortem index of serotonin turnover (5-HIAA/5-HT ratio) (Salome et al, 2006) in the amygdala, although whether this affects serotonin function per se remains unknown. In addition, trait anxiety is associated with altered structural and functional connectivity of the amygdala with the PFC (Grupe and Nitschke, 2013; Kim et al, 2011), and thus altered top-down control of the amygdala during emotion regulation. Of particular relevance are two medial regions, the ventromedial PFC (vmPFC, including area 14 and 25) and the dorsal anterior cingulate (dACC, anterior and posterior area 24) (Ongur and Price, 2000), which provide the most dense prefrontal output to the amygdala (Ghashghaei et al, 2007) and show, respectively, positive and negative functional coupling with it at rest (Roy et al, 2009). Attenuated vmPFC and exaggerated dACC activity at rest or in response to aversive stimuli (Kim et al, 2011; Sylvester et al, 2012), alongside dACC hypo-activation during emotion regulation (Blair et al, 2012; Sehlmeyer et al, 2011), is implicated in both trait and pathological anxiety. Thus, the present study also sought to determine whether trait-like anxiety in the marmoset is associated with (i) altered serotonergic function within the amygdala as measured by in vivo microdialysis and (ii) differences in brain volume in the amygdala, vmPFC, and dACC, using structural magnetic resonance imaging (MRI).

\section{MATERIALS AND METHODS}

\section{Subjects}

Thirty-nine adult common marmosets from the University of Cambridge Marmoset Breeding Colony (Callithrix jacchus, 20 females, 19 males, aged $2.4 \pm 0.5$ years) were used across Experiments 1, 2 and 3. In Experiment 1 (Figure 1a), all animals received the HI and model snake tests in order to characterise the high-anxiety phenotype. Of these, six low- and six high-anxious marmosets were used in Experiment 2, to investigate the attentional, cardiovascular and serotonergic correlates of trait-like anxiety (Figure $1 \mathrm{~b}$ ), and 23 marmosets were scanned with MRI in Experiment 3 (Figure 1c) to examine regional brain volume correlates of trait-like anxiety (Supplementary Materials and Methods 1).

Low- and high-anxious animals were identified according to the proportion of time spent in proximity to a $\mathrm{HI}$ (time spent at cage front, TSAF), a measure sensitive to anxiolytic drug treatment (Barros and Tomaz, 2002b). On the basis of the behavioral responses of a large cohort ( $n=63$, Agustin-Pavon et al., 2012) of experimentally native marmosets, $95 \%$ confidence intervals (CI) of the median were established, and TSAF scores $>25 \%$ (upper end point of the $95 \% \mathrm{CI}$ ) were defined as a low-anxiety phenotype, whereas TSAF scores $<9 \%$ (lower end point of the $95 \% \mathrm{CI}$ ) were defined as a high-anxiety phenotype (Supplementary Materials and Methods 1). All animals had minimal handling before the screening. Those animals from the large cohort destined for Experiments 1, 2 and 3 reported here had not been used in other experiments. All procedures were conducted in accordance with personal and project licenses held by the authors under the UK 1986 Animals (Scientific Procedures) Act.

\section{Experiment 1: Behavioral Characterisation of the High-Anxiety Phenotype}

Behavioral testing. Thirty-nine marmosets received the HI test (Agustin-Pavon et al., 2012; Figure 2a(i)) and model snake test (Shiba et al., 2014; Figure 2a(ii)) in the home cage (Supplementary Materials and Methods 2.1). Two smaller subgroups of animals were re-tested on the HI test at $\sim 6-$ 12 months $(n=14)$ and $2.5-3.5$ years $(n=12)$ (Figure 1a; Supplementary Materials and Methods 1).

\section{Data Analysis}

$\mathrm{HI}$ and snake tests were video-taped and scored for a range of behaviors and vocalisations (Figure 2a). Individual scores were standardised and used to calculate emotionality and coping strategy components of behavior using Principal Component Analysis (Supplementary Materials and Methods 5.1.1).

\section{Experiment 2: Relationship of Vigilance, Cardiovascular Reactivity and Amygdala Serotonin with a High-Anxiety Phenotype}

Behavioral testing. In the unpredictable threat test (Figure 3a; Supplementary Materials and Methods 2.2), marmosets first received five sessions of aversive contextual conditioning, comprising explicitly unpaired presentations of $105 \mathrm{~dB}$ aversive noise bursts and a neutral, $75 \mathrm{~dB}$ sound cue in an automated testing apparatus. To characterise conditioned responses to the context and the cue independently from one another, they were assessed during two probe sessions: (1) cue change: 12 presentations of a novel, neutral cue in the threatening context in the absence of aversive noise (Figure 3a(iii)) and (2) context change: 12 presentations of the cue originally had during conditioning, now within a novel, safe context (Figure 3a(iv)).

\section{Surgery}

Telemetry implantation. For the collection of cardiovascular data, animals were implanted with radiotelemetry transmitters (TA11PA-C40, Data Sciences) as described previously (Mikheenko et al, 2010).

Microdialysis. Extracellular levels of serotonin in the amygdala were measured by bilateral in vivo microdialysis under isoflurane anesthesia (Supplementary Materials and Methods 3). Microdialysis probes (MD-2200 Brain Microdialysis Probes, 2-mm membrane, BASi Instruments) were implanted at coordinates centered on the basal nucleus (AP $+9.3 \mathrm{~mm}, \mathrm{~L} \pm 5.6 \mathrm{~mm}, \mathrm{DV}+4.0 \mathrm{~mm}$ ). Following equilibration, three baseline samples were collected, and then 
Human intruder test

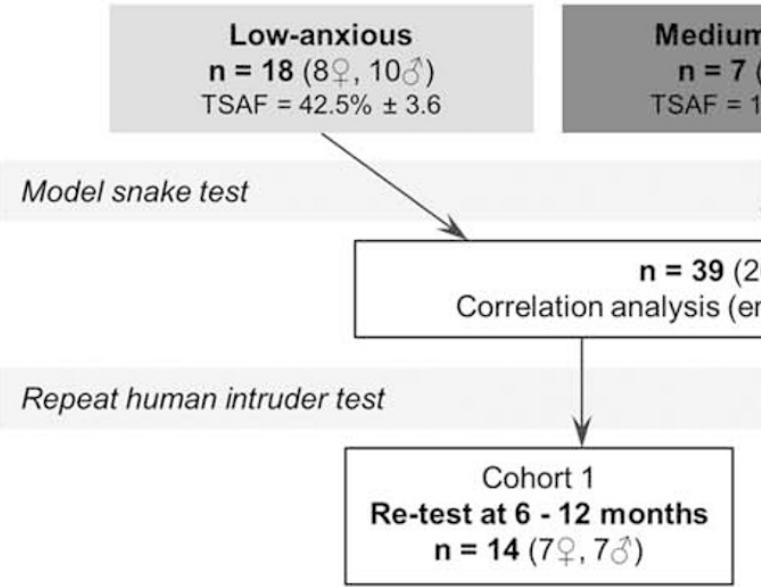

Medium-anxious

$\mathrm{n}=7(5+28)$

$\mathrm{SAF}=15.7 \% \pm 4.0$

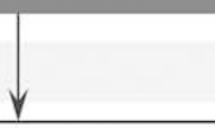

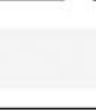

190

ty component)

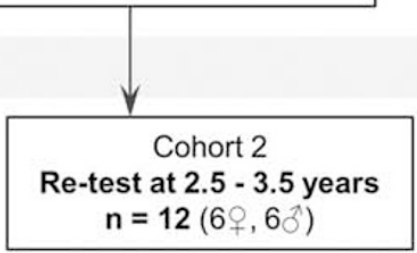

b Experiment 2. Relationship of vigilance, cardiovascular reactivity and amygdala serotonin with a high anxiety phenotype

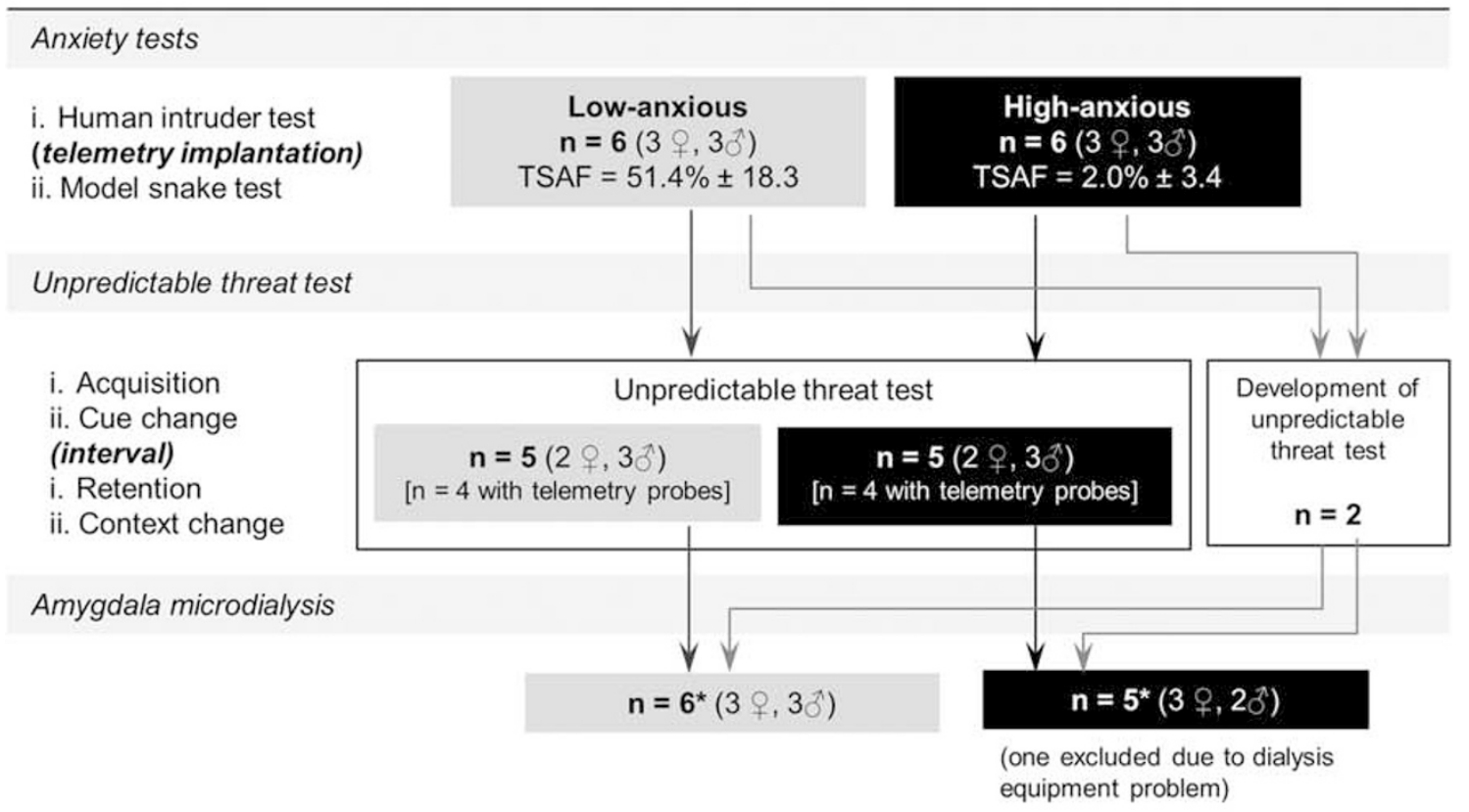

c Experiment 3. Relationship of brain structure with a high anxiety phenotype

Structural MRI imaging

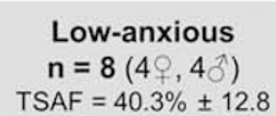

$\mathrm{n}=8(4 \%, 4 \hat{\jmath})$

TSAF $=40.3 \% \pm 12.8$
Medium-anxious

$\mathrm{n}=7\left(5 \%, 2 \delta^{2}\right)$

TSAF $=15.7 \% \pm 4.0$

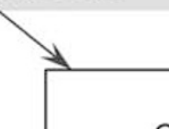

\section{TSAF $=15.7 \% \pm 4.0$}

$\mathbf{n}=\mathbf{2 3}(13$ 우 $10 \hat{)})$

Correlation analysis (TSAF vs. brain volume)
High-anxious

$\mathbf{n}=14\left(7 \%, 7 \delta^{\top}\right)$

TSAF $=3.0 \% \pm 1.0$ 


\section{a Methods: Home-cage anxiety tests}

\section{i. Human intruder test}

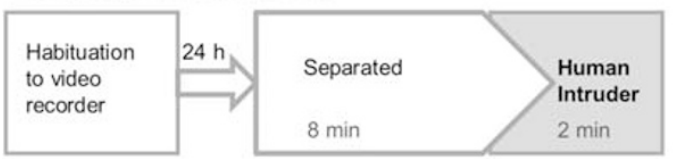

\section{PCA Measures}

Distance from cage front $(\mathrm{cm})$

Locomotion (proportion of time) and jumps (count)

Head-and-body bobbing (count)

Vocalisations (Tsik-egg, Tse, Tsik, Egg: count)

\section{ii. Model snake test}

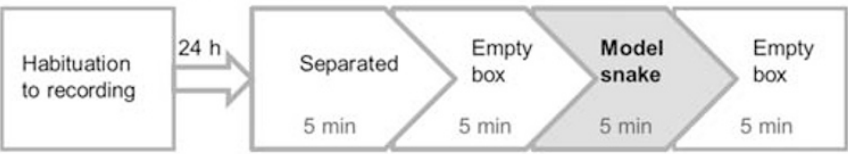

\section{PCA Measures}

Distance from cage front $(\mathrm{cm})$

Locomotion (proportion of time)

Looking (proportion of time)

Looks (count)

Head-cocking (count)

Vocalisations (Tsik, Tsik-egg: count)

\section{b Results: "Emotionality" component of responses in home-cage anxiety tests}
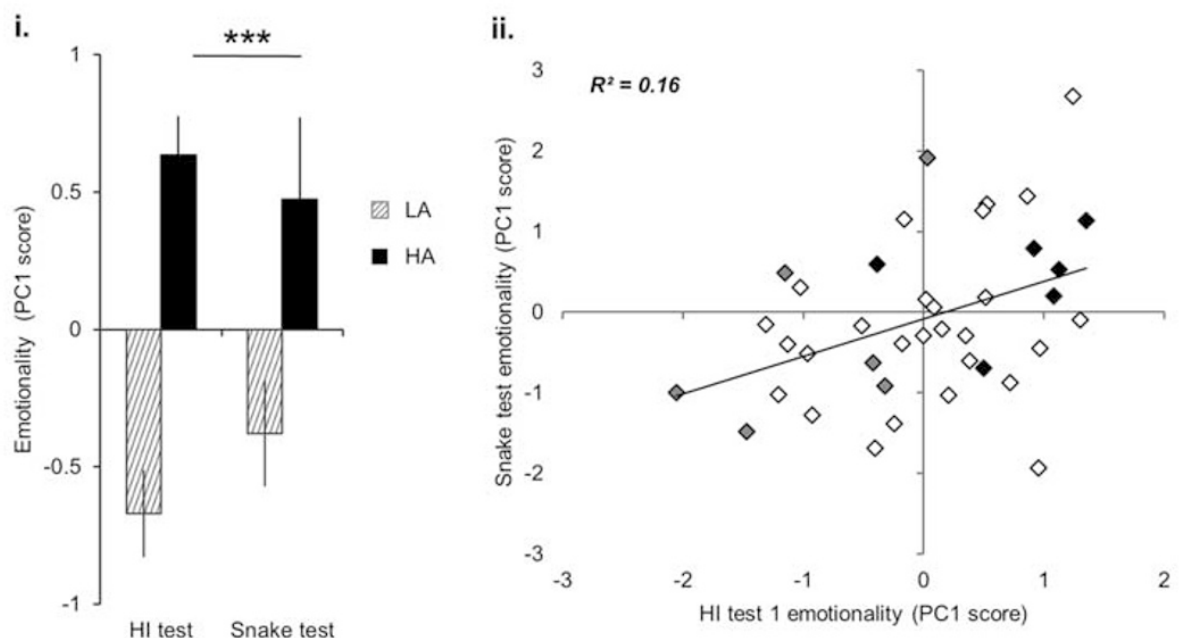

Figure 2 Home-cage anxiety tests. (a) Methods. (i) Human intruder test and (ii) model snake test were conducted in the home room, with the animal separated from its cage-mate and confined to the upper right-hand quadrant of the cage. Habituation was identical to the test session except that the intruder/snake was absent. All animals had at least a week between the tests. A principal component analysis (PCA) was performed on the behavior and vocalisation measures as detailed in (Agustin-Pavon et al, 20I2; Shiba et al, 20 I4) to calculate emotionality and coping strategy components of behavior in both tests. (b) Results. (i) Emotionality component of behavior in the initial human intruder test and model snake test in high- and low-anxious marmosets selected according to their 'time spent at cage front' scores. $* * * * P<0.001$ (ii) Higher emotionality during the initial human intruder test is correlated with significantly higher emotionality in response to the snake. Symbols shaded gray and black represent low and high trait-anxious marmosets, respectively, that went on to receive the unpredictable threat test and amygdala microdialysis in Experiment 2.

$1 \mu \mathrm{m}$ citalopram hydrobromide (Abcam Biochemicals, UK) was perfused through the probes for the remaining six samples. Animals had at least 2 weeks of recovery before behavioral testing re-commenced.

\section{Data Analysis}

Behavior. Video recordings were scored for the duration of active vigilance during the cue and pre-cue baseline (Figure 3a; Supplementary Materials and Methods 5.1.2).

Heart rate. Cardiovascular responses were monitored remotely using a PhysioTel Telemetry System (Data Sciences). HR was derived from the time interval between systolic blood pressure events (Supplementary Materials and Methods 5.2). Mean HR was calculated for the cue and pre-cue baseline periods.
Neurochemistry. Neurochemical content of microdialysis samples was analyzed by reversed-phase high-performance liquid chromatography (HPLC). Locations of microdialysis probe tracts were examined in two marmosets that died unexpectedly following microdialysis (Supplementary Materials and Methods 5.3-5.4).

\section{Experiment 3: Relationship of Brain Structure with a High-Anxiety Phenotype}

Twenty-three marmosets were scanned with MRI using a rapid acquisition with relaxation enhancement sequence at 4.7 $\mathrm{T}$ with a Bruker PharmaScan $47 / 16$ system at $250 \mu \mathrm{m}$ resolution. Standard procedures for anesthesia and monitoring were followed and are described with full MRI parameters in Supplementary Materials and Methods 4 .

Images were aligned together and a study-specific template for automated whole-brain analysis was produced 
a Methods: Unpredictable threat test

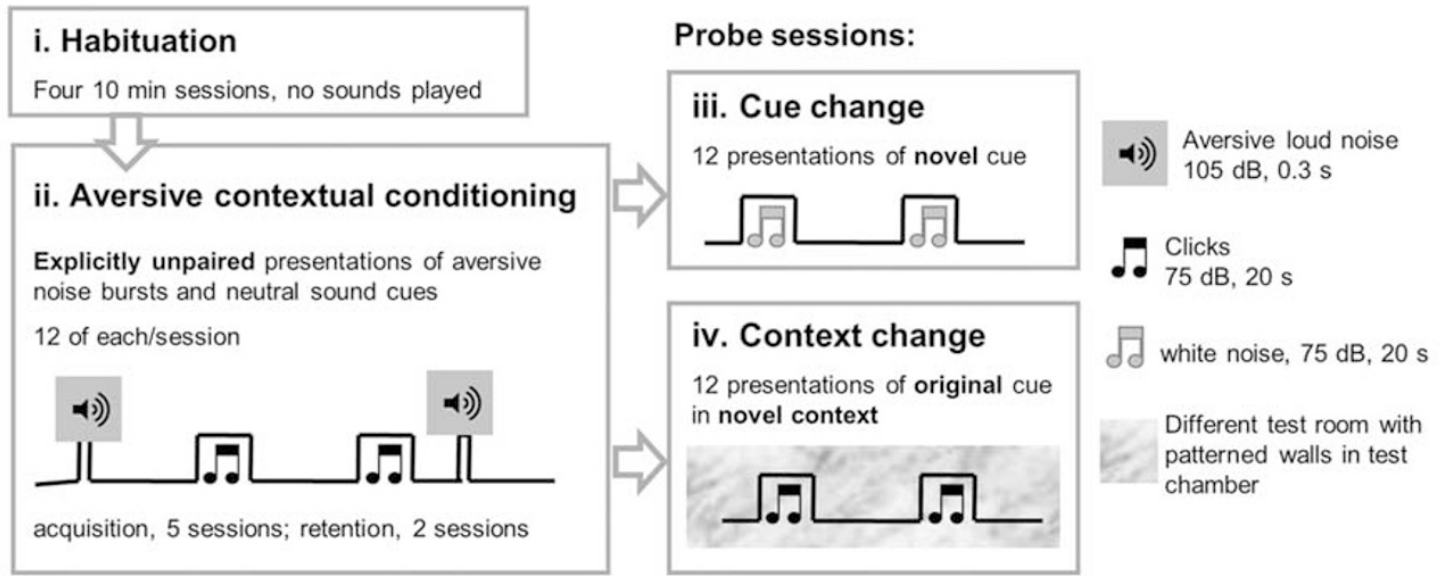

\section{b Results: Vigilance and HR in the context associated with unpredictable threat}

i.

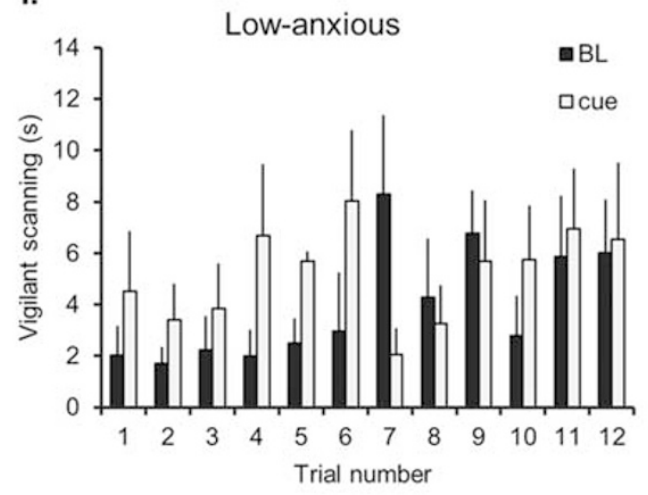

iii.

\begin{tabular}{lc} 
& $\begin{array}{c}\text { mean cue-BL } \\
\text { vigilance }(\mathbf{s})\end{array}$ \\
\hline LA1 & $0.46 \pm 1.28$ \\
LA2 & $0.01 \pm 1.71$ \\
LA3 & $1.66 \pm 0.82$ \\
LA4 & $2.86 \pm 2.35$ \\
\hline HA1 & $5.52^{\star \star} \pm 1.40$ \\
HA2 & $6.83^{\star \star} \pm 1.45$ \\
HA3 & $\mathbf{1 . 8 7}^{\star \star} \pm 0.59$ \\
HA4 & $2.97^{\star} \pm 0.97$ \\
\hline
\end{tabular}

iv.

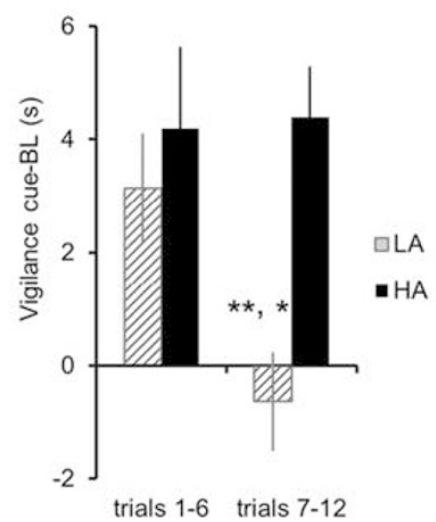

ii.

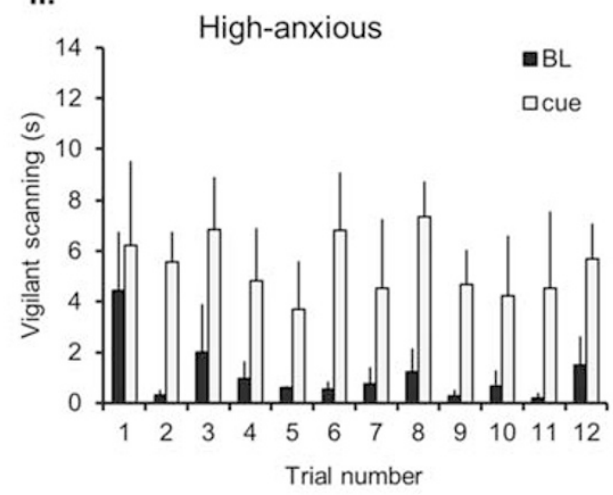

v.

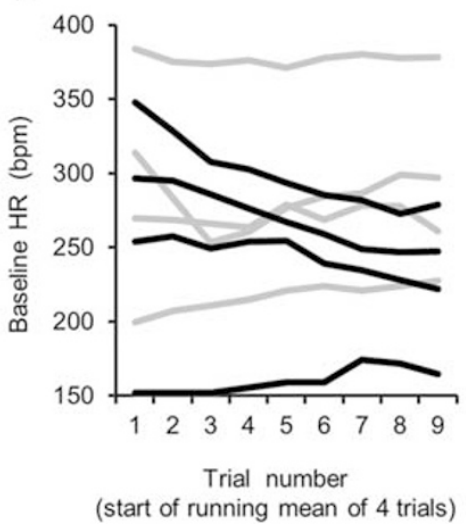

Figure 3 Unpredictable threat test. (a) Methods. Vigilant scanning behavior (active visual search of the surroundings, accompanied by tense postures marked by forward extension of body and/or head and rearing (Mikheenko et al, 20 I0; Shiba et al, 20 I4)) was assessed in the unpredictable threat test, as duration of the behavior over 20-s cue and 20-s baseline (pre-cue) periods. (i) Habituation consisted of four I0-min sessions with no sounds presented. (ii) Acquisition and retention of aversive contextual conditioning comprised daily 30-min sessions. The volume of the aversive noise was sufficient to elicit a bodily startle response, but was somewhat lower than that used in other studies (I I6-120 dB, Shiba et al 20 I4, Zeredo et al, 20 I4) in order to ensure that high-anxious animals would still voluntarily enter the carry box. Inter-stimulus interval $=40-80 \mathrm{~s}$. (iii) Acquisition was followed by the presentation of a neutral, novel cue in the context associated with unpredictable aversive noise (cue change) but in the absence of the aversive noise. Length of session $\sim 30 \mathrm{~min}$; inter-stimulus interval $=40-160 \mathrm{~s}$. (iv) Cue change was followed by retention and a context change session, in which the marmoset was placed into a novel, visually distinct context and presented with the neutral, familiar cue that had been played during acquisition/retention, again in the absence of aversive noise. Length of session $\sim 30$ min; inter-stimulus interval $=40-160 \mathrm{~s}$. (b) Results. (i) Vigilance in the threatening context, low-anxious group. (ii) Vigilance in the threatening context, high-anxious group. (iii) Individual cue-specific vigilance scores (mean across the session), *P $<0.05$, $* * P<0.01$ Is baseline. (iv) Cue-specific vigilance changes in first vs second half of the session, $* P<0.05$ vs LA trials $I-6$; $* * *<0.0$ I vs HA trials $7-12$. (v) Baseline HR in the threatening context. Black lines: high-anxious, gray lines: low-anxious animals. 
following (Sawiak et al, 2009) (Supplementary Materials and Methods 5.5). Regions of interest (ROIs) corresponding to the dACC, vmPFC and amygdala were manually drawn on this template using Analyze 8.1 (Mayo Clinic) according to the Paxinos et al (2011) marmoset atlas. We performed whole-brain automated voxel-based tests with tensor-based morphometry (TBM) to find voxels with localised volume changes that correlated with TSAF, in addition to manual volumetry by region tracing. To conduct TBM, Jacobian determinants (maps of local volume change) for each brain were smoothed with an $800 \mu \mathrm{m}^{3}$ isotropic Gaussian smoothing kernel. Gender and overall brain volume were included as covariates in the general linear model. In an exploratory whole-brain analysis, type I errors owing to multiple comparisons were mitigated using a reduced $P$-value of $P<0.001$, with a cluster extent threshold of 50 voxels. If voxels were found in the three key areas of interest, a smallvolume correction was applied using the ROIs. Random field theory was used within such regions to control the family-wise error rate with $P_{\mathrm{FWE}}<0.05$. Manually-traced ROIs from the template were analyzed by extracting the mean Jacobian determinant throughout each region to measure regional volumes in each subject.

\section{Statistics}

Statistical analysis was performed in SPSS 22.0. The data were checked for normality and homoscedasticity, and one-sample $t$-tests, ANOVA with Sidak or Fisher's LSD pair-wise comparisons, or non-parametric Mann-Whitney tests, were used as appropriate. Statistical significance was set at $P \leq 0.05$. Correlations were quantified with Pearson's correlation coefficient.

\section{RESULTS}

\section{Experiment 1}

Persistent emotional responses to a human intruder and model snake reflect trait-like anxiety. Marmosets that spent $<9 \%$ of their time in proximity to a HI showed a behavioral profile consistent with high anxiety, including a greater average distance from the intruder, fewer jumps towards the intruder and increased headand-body bobbing compared with the low-anxious group ( $>25 \%$ TSAF) (Supplementary Results 1.1, Supplementary Table S1). They also showed more anxious responses to a model snake, including significant avoidance of visual contact with it (Supplementary Results 1.2, Supplementary Table S2). Analysis of the overall emotionality scores revealed that high-anxious animals showed significantly higher emotionality across both tests: Figure $2 \mathrm{~b}(\mathrm{i})$, Repeated measures ANOVA, main effects of group: $\mathrm{F}_{(1,30)}=20.4, P<0.001$; test: $\mathrm{F}_{(1,30)}<1$, test $\times$ group interaction $\mathrm{F}_{(1,30)}=2.00$, both NS. A correlation analysis including a further seven medium-anxious animals confirmed that heightened emotionality toward the intruder is associated with greater emotionality toward the snake Figure $2 \mathrm{~b}$ (ii), $\left.r_{(37)}=0.40, P<0.05\right)$, suggesting that performance on both tests reflects a common underlying anxiety-like trait.

Analysis of emotionality scores for HI re-test in two separate subgroups of the original cohort, at $\sim 6-12$ months
( $n=14)$ and 2.5-3.5 years $(n=12)$, confirmed that the traitlike anxiety responses to an intruder are stable over time (Supplementary Results 1.3, Supplementary Figure S1).

\section{Experiment 2}

High-anxious marmosets show sustained vigilance to a novel cue in a threatening context. High- and lowanxious marmosets did not differ in their responses during contextual conditioning (Supplementary Results 2). However, marked differences in vigilance emerged when the marmosets were re-exposed to the threatening context a week later and presented with a novel, neutral cue in the absence of aversive noise. Low-anxious marmosets showed a transient vigilant response directed specifically to the novel cue, followed by a general increase in vigilance during both cue and baseline periods (Figure 3b(i)). In contrast, high-anxious marmosets maintained high cue-specific vigilance across the entire session, with very low responses to the context alone (Figure $3 \mathrm{~b}$ (ii)). Individually, every high-anxious animal, but no low-anxious animal, showed a significant increase in vigilance to the cue compared with baseline (Figure 3b(iii)).

An ANOVA of cue responses relative to baseline revealed a significant group $\times$ time interaction, such that only lowanxious marmosets showed a decline in cue-specific vigilance in the second half of the session, relative to their own responding in the first half, as well as to that of the high-anxious group (Figure 3b(iv); group $\times$ time interaction: $\left.\mathrm{F}_{(1,6)}=6.67, P<0.05\right)$. A similar comparison of vigilance responses during the baseline (indicative of responses to the overall context) showed that only the low-anxious group showed heightened vigilance in the second half of the session, likely reflecting growing anticipation of the aversive noise that had not yet come. The high-anxious group, on the other hand, showed a sustained response bias toward the cue, even though it was not predictive of threat, throughout the session. The same pattern of results was observed when the two marmosets that underwent behavioral testing but were not implanted with telemetry probes were included in the analysis (Supplementary Results 3.1). No differences were observed between the groups in a novel, safe context (Supplementary Results 4).

Three out of four high-anxious animals showed a marked reduction in baseline HR during exposure to the threatening context, unlike low-anxious animals (Figure $3 b(v)$ ). However, group differences did not reach significance, and HR responses to the cues were likewise unaffected by trait anxiety (Supplementary Results 3.2).

High-anxious marmosets show reduced extracellular serotonin in the amygdala. In vivo microdialysis in the amygdala (Figure $4(\mathrm{a})$ ) revealed significantly lower extracellular serotonin in high-anxious animals, following infusion of the selective serotonin reuptake inhibitor citalopram (Figure 4(b); high-anxious levels expressed as $\%$ of low-anxious mean, one-sample $t$-tests with Sidak correction, samples 4-6: $t_{(4)}=-4.87, P<0.05$, samples 7-9: $\left.t_{(4)}=-6.19, P<0.01\right)$. The groups did not differ in 5-HIAA levels or in serotonin turnover rate (5-HIAA/5-HT ratio) (Supplementary Results 5). 


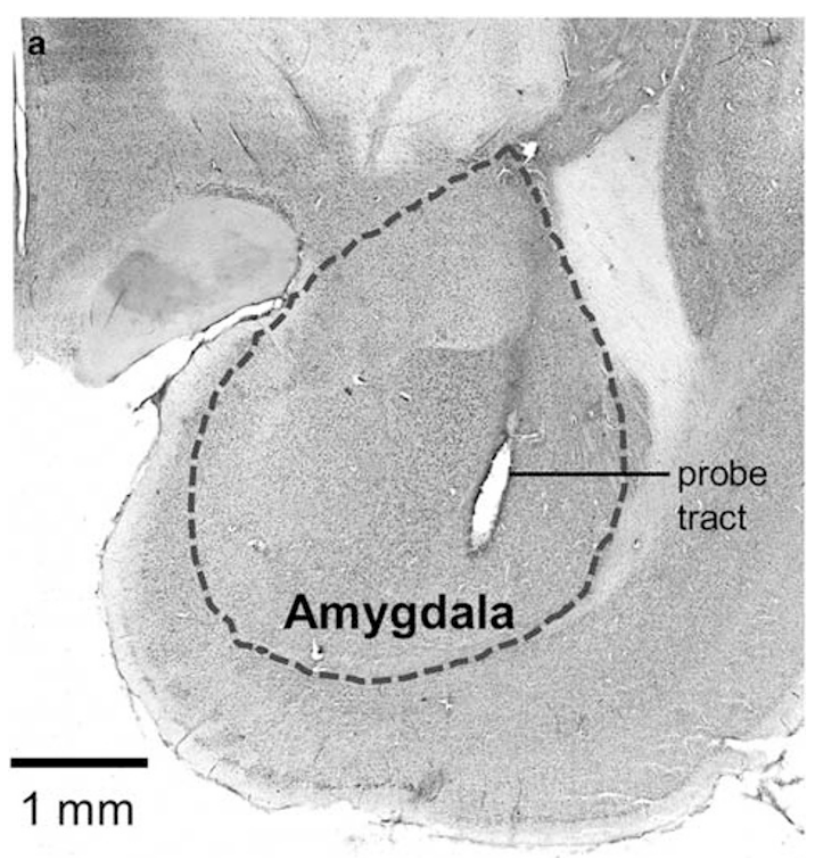

b

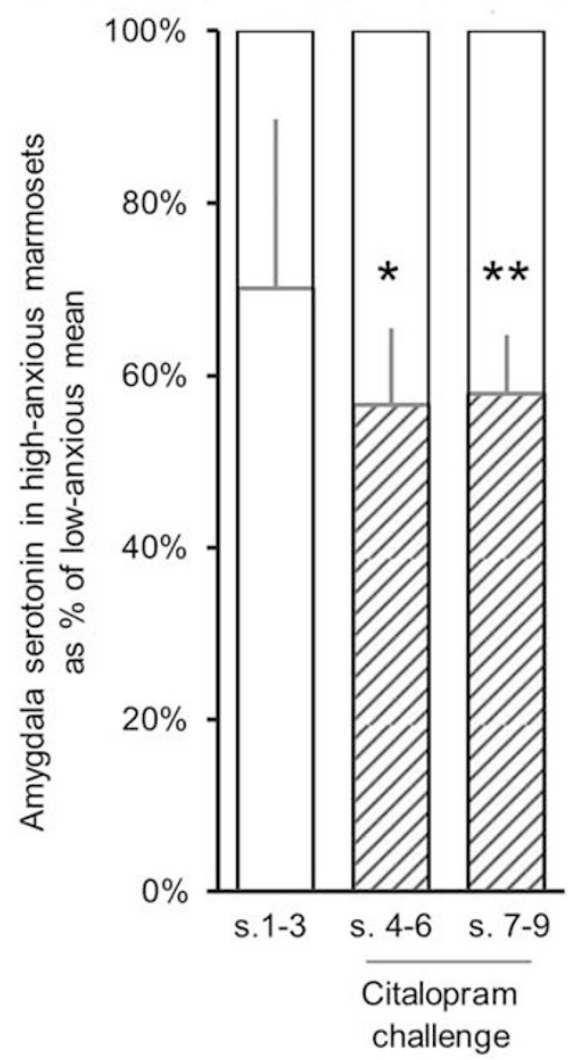

Figure 4 Amygdala microdialysis (in vivo extracellular serotonin levels). (a) Coronal section through the amygdala illustrating the positioning of the microdialysis probe. (b) Extracellular levels of amygdala serotonin in highanxious marmosets expressed as \% of the mean low-anxious levels, at baseline (white bars, samples I-3, no citalopram) and following citalopram challenge (shaded bars, samples 4-6 and samples 7-9). ${ }^{*} P<0.05$, ** $P<0.0$ l compared with low-anxious mean. Although three out of five high-anxious animals also showed substantially reduced serotonin levels at baseline (39-52\% of low-anxious mean), this did not reach statistical significance for the group (samples $1-3, t_{(4)}=-1.53, P=0.49$ ).

\section{Experiment 3}

Higher trait-like anxiety is associated with reduced dACC volume. Tensor-based morphometry results are shown in Figure 5(a). Whole-brain exploratory analysis revealed that the most significant cluster was within the dACC $(P<0.05$, corrected), where smaller brain volume was associated with more anxious behavior (lower TSAF): $r=0.80$, Figure $5(\mathrm{~b})$, with an additional cluster in the posterior cingulate cortex $(P<0.001$ uncorrected $)$.

Correlational analysis was also performed on the ROI volumes for the dACC, vmPFC and amygdala with TSAF. Initial analysis revealed a significant positive correlation in the right dACC $\left(r_{(21)}=0.47, P=0.025\right)$, left dACC $\left(r_{(21)}=\right.$ $0.43, P=0.043)$, and also in the right $\operatorname{vmPFC}\left(r_{(21)}=0.43\right.$, $P=0.039)$. A similar correlation was found in both prefrontal regions when left and right volumes were averaged (Supplementary Results 6). However, none of these individually significant correlations survived the Sidak correction for multiple comparisons.

\section{DISCCUSSION}

A high-anxious behavioral phenotype in marmosets was identified as persistent, trait-like emotional responses to both a human intruder and a model snake, and sustained vigilance to novel, neutral cues in a context associated with unpredictable threat. In vivo microdialysis in the amygdala revealed lower levels of serotonin, and structural MRI revealed reduced volume within the AACC of high-anxious compared with low-anxious marmosets.

High-anxious marmosets, identified according to the extent of their withdrawal from the cage front when stared at by a human intruder, showed stable, trait-like emotionality in two standard tests of anxiety, ie, exposure to a human intruder and a model snake. We suggest that such trait-like anxiety in the marmoset may share important similarities with anxious temperament in other primates, eg, (Fox and Kalin, 2014; Suomi, 1997). A prominent series of studies in rhesus monkeys (reviewed by Fox and Kalin, 2014) defines anxious temperament behaviorally as heightened freezing and vigilance when the monkey is confronted by a human intruder. These behaviors are most prominent when the intruder presents their profile to the monkey while avoiding eye contact, eg, (Corcoran et al, 2012; Rogers et al, 2008), but freezing has also been reported in response to direct stare from the intruder (Corcoran et al, 2012; Machado and Bachevalier, 2008). We did not observe freezing in our marmosets; however the overall pattern of withdrawal from the intruder and increased head-and-body bobbing/swaying (an alarm behavior (Barros et al, 2002a) accompanied by vigilance-related egg calls) in high-anxious marmosets is somewhat similar to the combination of behavioral inhibition (eg, freezing) and hyper-vigilance in rhesus monkeys with anxious temperament. Both of these behavior styles have also been noted in persistent anxiety associated with social subordination stress (Shively and Willard, 2012) or early life stress (Corcoran et al, 2012; Spinelli et al, 2009) in macaques; to what extent this may be related to trait-like anxiety in the marmoset remains a question for further study. 
a

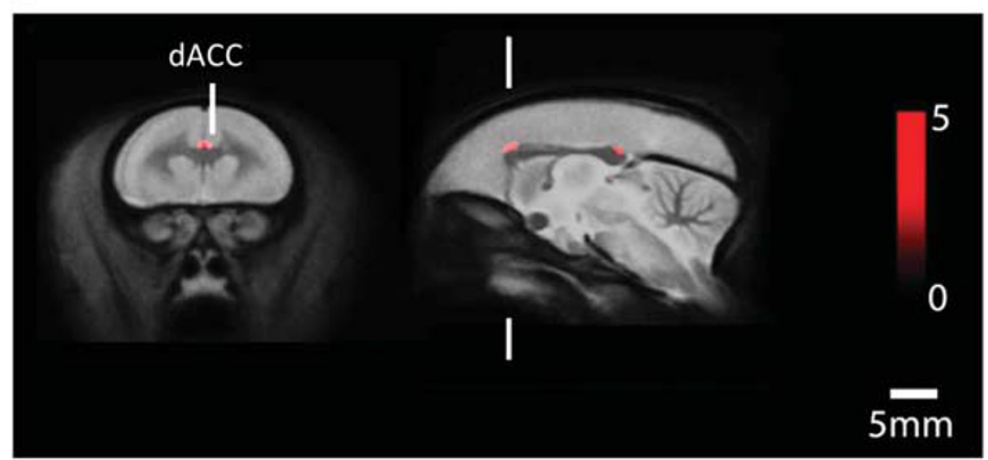

b

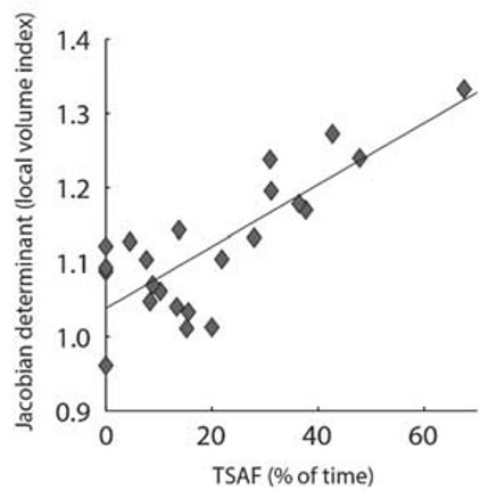

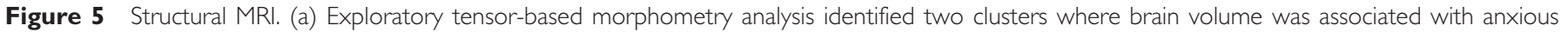

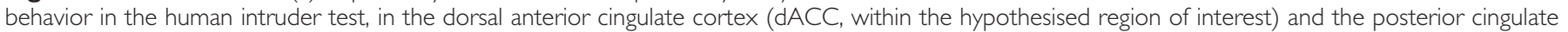

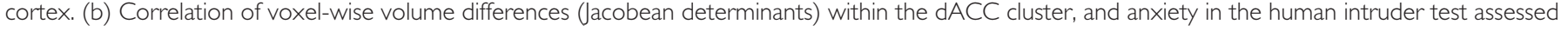
by the $\%$ of time spent at cage front (TSAF) in the presence of the intruder.

Alongside heightened emotionality in the home-cage tests, high-anxious marmosets showed sustained vigilance to abstract, neutral cues presented in a context associated with unpredictable threat. This may reflect either heightened attentional capture by a salient stimulus within a threatening context (prior to appraisal of the cue as safe or threatening), or biased appraisal of an ambiguous cue as threatening. With respect to the former, accounts of attentional and cognitive control in humans (Braver, 2012; Eysenck et al, 2007) propose that anxiety increases the influence of the stimulus-driven attentional system, resulting in an exaggerated or inflexible focus on salient cues. This may be heightened in trait-anxious individuals, leading to accentuated responses to neutral cues in a threatening context both in marmosets (this study) and people (Mikheenko et al, 2014). On the other hand, a novel cue introduced into a context of unpredictable threat may be initially perceived as ambiguous (it being unclear, at first, whether it may be a better predictor of threat), and thus appraised by high-anxious individuals as threatening (Grupe and Nitschke, 2013). In this case, the heightened cue vigilance here may be similar to the undifferentiated vigilance shown by high-anxious marmosets to explicit threat and safety cues (Shiba et al, 2014). Of note, the low-anxious marmosets that showed a rapid habituation of cue vigilance in this study subsequently showed sustained cue vigilance following selective depletion of serotonin in the ventral PFC (orbitofrontal cortex and frontal pole, (Mikheenko et al, 2012)), underscoring the importance of prefrontal regulation of responses in a threatening context (Agustin-Pavon et al, 2012; Braver, 2012, Grupe and Nitschke, 2013).

In contrast to their sustained cue-directed vigilance, highanxious marmosets showed unusually low levels of vigilance in the baseline (no-cue) periods, together with a decrease in baseline HR over the session in three out of four animals. This was often accompanied by generally reduced movement, huddled posture and a notable absence of selfgrooming and box-directed behaviors. Speculatively, this may reflect a withdrawal response under stress in the absence of salient cues to trigger attention. Of note, in a more intensely aversive context, some high-anxious marmosets show a still more pervasive withdrawal, with virtually no behavioral response and a marked bradycardia to novel cues (Zeredo et al, 2014), similar to that described by Porges (1985). This combination of hyper-vigilance and withdrawal has also been noted in stress-related depression in macaque monkeys (Shively and Willard, 2012), and may shed light on the contribution of trait anxiety to the development of depression (Sandi and Richter-Levin, 2009).

Within the amygdala, high-anxious marmosets showed similar structural volume but reduced extracellular serotonin levels compared with their low-anxious counterparts. Tonic action of serotonin via 5-HT2a receptors is thought to facilitate GABAergic inhibition within the amygdala (Jiang et al, 2009). Thus, lower serotonin levels in high-anxious individuals may sensitize the amygdala to sensory input and allow innocuous cues to be processed as threatening, triggering heightened vigilance. This is consistent with recent proposals linking low tonic serotonin and amygdala hyperactivity in individuals with the short allele of 5-HTT (Jasinska et al, 2012) or with increased 5-HTT promoter methylation (Nikolova et al, 2014). A reduction in tonic serotonin signaling may also amplify phasic (5-HT2c-mediated) responses to aversive events (Cools et al, 2008), further contributing to heightened anxiety. High-anxious individuals may also show global alterations in serotonin function across a number of anxiety-associated brain regions eg, BNST, hippocampus, and mPFC (Oler et al, 2009; Yokoyama et al, 2013).

Given that genetic and epigenetic variability in the 5-HTT promoter region affects amygdala reactivity to threat (Heinz et al, 2005; Nikolova et al, 2014) and amygdala-vmPFC interactions (Heinz et al, 2005), it would be insightful to explore similar effects in the marmoset. Whereas there is no published evidence for variation in the marmoset 5-HTT promoter region (Pascale et al, 2012), a putatively functional polymorphism has been identified in our laboratory (Santangelo, Roberts et al, unpublished data) and in this study low- and high-anxious groups were counterbalanced for this genotype.

Structural imaging revealed that trait-like anxiety in the marmosets is associated with altered volume in a prefrontal region closely connected with the amygdala, the dACC. Those animals that spent the majority of their time away from the human intruder, and thus were the more anxious, 
had the smallest dACC volumes. This is consistent with reports of reduced metabolic activity in the dACC in rhesus monkeys that show poorer contextual regulation of freezing in the HI test (Kalin et al, 2005), as well as reduced dACC volume (Asami et al, 2008; Radua et al, 2010) and activity during emotion regulation (Blair et al, 2012) in anxiety disorders. However, it differs from other reports of hyperactivation in the dACC related to chronic anxiety in humans (Kim et al, 2011; Sylvester et al, 2012), and enlarged dACC in peer-reared rhesus monkeys showing persistent anxiety (Spinelli et al, 2009). These apparently opposing effects may be a consequence of the heterogeneity of functioning along the rostro-caudal extent of the dACC, with more rostral regions being associated with appraisal and anticipation of threat, processing of uncertainty (Grupe and Nitschke, 2013; Kalisch and Gerlicher, 2014), conscious emotion regulation (Giuliani et al, 2011), and effortful control of attention. In contrast, more caudal regions are associated with fear expression (Milad and Quirk, 2012), including the generation and interoceptive awareness of accompanying autonomic arousal. It is of note that the change in dACC volume in the present study was relatively rostral, at the level of the genu of the corpus callosum, and that functional connectivity and serotonin transporter binding within this region in the marmoset has been previously associated with responses to unfamiliar conspecifics (and, specifically, anxious behaviors) (Yokoyama et al, 2013). Further studies involving targeted manipulation of the rostral and caudal dACC may help unravel the complex role of this region in trait-like and pathological anxiety.

Voxel-based morphometry has a number of limitations that should be highlighted. Principally, the method assumes that coregistration between homologous voxels is accurate and voxel-wise statistical analysis relies upon this. We have mitigated this to some extent by smoothing the data, and by manually inspecting the results of image registration, and we did not see any obvious problems. The absence of significant voxels in a region does not prove it is unaffected, as our method relies on rejecting the null hypothesis at each voxel. Nevertheless, we do report the whole-brain exploratory analysis (albeit without stringent control of type I errors), even though we had the clear objective of assessing specific brain regions for differences. The secondary area found in the exploratory analysis, the posterior cingulate cortex, was not assessed further as we did not have an $a$ priori hypothesis. We note, however, that the region has been implicated in human anxiety (Sylvester et al, 2012) and warrants further analysis in future studies, alongside other cortical areas associated with trait anxiety and threat responsiveness, such as the insula, orbitofrontal cortex, and ventrolateral PFC (Agustin-Pavon et al, 2012; Grupe and Nitschke, 2013; Sylvester et al, 2012).

In summary, high-anxious marmosets showed greater emotionality in home-cage anxiety tests, and sustained attention to neutral cues in a context associated with unpredictable threat. Neurally, high trait-like anxiety was associated with reduced in vivo amygdala serotonin and a smaller dACC volume. Together, these observations highlight the marked similarity in the physiological and behavioral characteristics of trait anxiety in monkeys and humans, and lay the foundation for further studies of the neural and genetic underpinnings of anxiety.

\section{FUNDING AND DISCLOSURE}

This research was supported by a Medical Research Programme Grant (G0901884) from the Medical Research Council UK (MRC) to Angela Roberts, and a PhD studentship from MRC and final-term funding from Trinity College, Cambridge, UK to Yevheniia Mikheenko. The authors declare no conflict of interest.

\section{ACKNOWLEDGEMENTS}

We wish to thank J Xia for HPLC analysis of microdialysis samples and preparation of aCSF, M Arroyo for histological preparation, A Santangelo for genotyping and assistance with human intruder testing, and $\mathrm{N}$ Horst and C Kim for assistance with experimental procedures.

\section{REFERENCES}

Agustin-Pavon C, Braesicke K, Shiba Y, Santangelo AM, Mikheenko Y, Cockroft G et al (2012). Lesions of ventrolateral prefrontal or anterior orbitofrontal cortex in primates heighten negative emotion. Biol Psychiatry 72: 266-272.

Asami T, Hayano F, Nakamura M, Yamasue H, Uehara K, Otsuka T et al (2008). Anterior cingulate cortex volume reduction in patients with panic disorder. Psychiatry Clin Neurosci 62: 322-330.

Barros M, Boere V, Mello E Jr, Tomaz C (2002a). Reactions to potential predators in captive-born marmosets (Callithrix penicillata). Int J Primatol 23: 443-454.

Barros M, Tomaz C (2002b). Non-human primate models for investigating fear and anxiety. Neurosci Biobehav Rev 26: 187-201.

Blair KS, Geraci M, Smith BW, Hollon N, DeVido J, Otero M et al (2012). Reduced dorsal anterior cingulate cortical activity during emotional regulation and top-down attentional control in generalized social phobia, generalized anxiety disorder, and comorbid generalized social phobia/generalized anxiety disorder. Biol Psychiatry 72: 476-482.

Braver TS (2012). The variable nature of cognitive control: a dual mechanisms framework. Trends Cogn Sci 16: 106-113.

Cools R, Calder AJ, Lawrence AD, Clark L, Bullmore E, Robbins TW (2005). Individual differences in threat sensitivity predict serotonergic modulation of amygdala response to fearful faces. Psychopharmacology (Berl) 180: 670-679.

Cools R, Roberts AC, Robbins TW (2008). Serotoninergic regulation of emotional and behavioural control processes. Trends Cogn Sci 12: 31-40.

Corcoran CA, Pierre PJ, Haddad T, Bice C, Suomi SJ, Grant KA et al (2012). Long-term effects of differential early rearing in rhesus macaques: behavioral reactivity in adulthood. Dev Psychobiol 54: 546-555.

Davis M, Walker DL, Miles L, Grillon C (2010). Phasic vs sustained fear in rats and humans: role of the extended amygdala in fear vs anxiety. Neuropsychopharmacology 35: 105-135.

Eysenck MW, Derakshan N, Santos R, Calvo MG (2007). Anxiety and cognitive performance: attentional control theory. Emotion 7: 336-353.

Faria V, Ahs F, Appel L, Linnman C, Bani M, Bettica P et al (2014). Amygdala-frontal couplings characterizing SSRI and placebo response in social anxiety disorder. Int J Neuropsychopharmacol 17: 1149-1157.

Fox AS, Kalin NH (2014). A translational neuroscience approach to understanding the development of social anxiety disorder and Its pathophysiology. Am J Psychiatry 171: 1162-1173.

Ghashghaei HT, Hilgetag CC, Barbas H (2007). Sequence of information processing for emotions based on the anatomic dialogue between prefrontal cortex and amygdala. Neuroimage 34: 905-923. 
Giuliani NR, Drabant EM, Gross JJ (2011). Anterior cingulate cortex volume and emotion regulation: is bigger better? Biol Psychol 86: 379-382.

Grillon C, Pine DS, Lissek S, Rabin S, Bonne O, Vythilingam M (2009). Increased anxiety during anticipation of unpredictable aversive stimuli in posttraumatic stress disorder but not in generalized anxiety disorder. Biol Psychiatry 66: 47-53.

Grupe DW, Nitschke JB (2013). Uncertainty and anticipation in anxiety: an integrated neurobiological and psychological perspective. Nat Rev Neurosci 14: 488-501.

Heinz A, Braus DF, Smolka MN, Wrase J, Puls I, Hermann D et al (2005). Amygdala-prefrontal coupling depends on a genetic variation of the serotonin transporter. Nat Neurosci 8: 20-21.

Jasinska AJ, Lowry CA, Burmeister M (2012). Serotonin transporter gene, stress and raphe-raphe interactions: a molecular mechanism of depression. Trends Neurosci 35: 395-402.

Jiang X, Xing G, Yang C, Verma A, Zhang L, Li H (2009). Stress impairs 5-HT2A receptor-mediated serotonergic facilitation of GABA release in juvenile rat basolateral amygdala. Neuropsychopharmacology 34: 410-423.

Kalin NH, Shelton SE, Fox AS, Oakes TR, Davidson RJ (2005). Brain regions associated with the expression and contextual regulation of anxiety in primates. Biol Psychiatry 58: 796-804.

Kalisch R, Gerlicher AMV (2014). Making a mountain out of a molehill: on the role of the rostral dorsal anterior cingulate and dorsomedial prefrontal cortex in conscious threat appraisal, catastrophizing, and worrying. Neurosc Biobehav Rev 42: 1-8.

Kim MJ, Loucks RA, Palmer AL, Brown AC, Solomon KM, Marchante AN et al (2011). The structural and functional connectivity of the amygdala: from normal emotion to pathological anxiety. Behav Brain Res 223: 403-410.

Machado CJ, Bachevalier J (2008). Behavioral and hormonal reactivity to threat: effects of selective amygdala, hippocampal or orbital frontal lesions in monkeys. Psychoneuroendocrinology 33: 926-941.

Mikheenko Y, Braesicke K, Xia J, Clarke H, Roberts AC (2012). Individual differences in trait anxiety affect vigilance during unpredictable threat: contribution of serotonin in the ventral prefrontal cortex. 2012 Neuroscience Meeting Planner Program No. 422.06. Online.

Mikheenko Y, Fletcher D, Roberts AC, Clark L (2014). Undifferentiated physiological responses to safety and unpredictable threat are associated with high trait anxiety and lower emotional resilience in competitive sport. 2014 Neuroscience Meeting Planner Program No. 470.13. Online.

Mikheenko Y, Man MS, Braesicke K, Johns ME, Hill G, Agustin-Pavon C et al (2010). Autonomic, behavioral, and neural analyses of mild conditioned negative affect in marmosets. Behav Neurosci 124: 192-203.

Milad MR, Quirk GJ (2012). Fear extinction as a model for translational neuroscience: ten years of progress. Annu Rev Psychol 63: 129-151.

Nikolova YS, Koenen KC, Galea S, Wang CM, Seney ML, Sibille E et al (2014). Beyond genotype: serotonin transporter epigenetic modification predicts human brain function. Nat Neurosci 17: $1153-1155$.

Oler JA, Fox AS, Shelton SE, Christian BT, Murali D, Oakes TR et al (2009). Serotonin transporter availability in the amygdala and bed nucleus of the stria terminalis predicts anxious temperament and brain glucose metabolic activity. J Neurosci 29: 9961-9966.
Oler JA, Fox AS, Shelton SE, Rogers J, Dyer TD, Davidson RJ et al (2010). Amygdalar and hippocampal substrates of anxious temperament differ in their heritability. Nature 466: 864-868.

Ongur D, Price JL (2000). The organization of networks within the orbital and medial prefrontal cortex of rats, monkeys and humans. Cereb Cortex 10: 206-219.

Pascale E, Lucarelli M, Passarelli F, Butler RH, Tamellini A, Addessi $\mathrm{E}$ et al (2012). Monomorphic region of the serotonin transporter promoter gene in New World monkeys. Am J Primatol 74: 1028-1034.

Paxinos G, Watson C, Petrides M, Rosa MG, Tokuno H (2011). The Marmoset Brain in Stereotaxic Coordinates. Academic Press Inc: San Diego324.

Radua J, van den Heuvel OA, Surguladze S, Mataix-Cols D (2010). Meta-analytical comparison of voxel-based morphometry studies in obsessive-compulsive disorder vs other anxiety disorders. Arch Gen Psychiatry 67: 701-711.

Rogers J, Shelton SE, Shelledy W, Garcia R, Kalin NH (2008). Genetic influences on behavioral inhibition and anxiety in juvenile rhesus macaques. Genes Brain Behav 7: 463-469.

Roy AK, Shehzad Z, Margulies DS, Kelly AM, Uddin LQ, Gotimer K et al (2009). Functional connectivity of the human amygdala using resting state fMRI. Neuroimage 45: 614-626.

Salome N, Viltart O, Lesage J, Landgraf R, Vieau D, Laborie C (2006). Altered hypothalamo-pituitary-adrenal and sympathoadrenomedullary activities in rats bred for high anxiety: central and peripheral correlates. Psychoneuroendocrinology 31: 724-735.

Sandi C, Richter-Levin G (2009). From high anxiety trait to depression: a neurocognitive hypothesis. Trends Neurosci 32: 312-320.

Sawiak SJ, Wood NI, Williams GB, Morton AJ, Carpenter TA (2009). Voxel-based morphometry in the R6/2 transgenic mouse reveals differences between genotypes not seen with manual $2 \mathrm{D}$ morphometry. Neurobiol Dis 33: 20-27.

Sehlmeyer C, Dannlowski U, Schoning S, Kugel H, Pyka M, Pfleiderer B et al (2011). Neural correlates of trait anxiety in fear extinction. Psychol Med 41: 789-798.

Shiba Y, Santangelo AM, Braesicke K, Agustin-Pavon C, Cockcroft G, Haggard $\mathrm{M}$ et al (2014). Individual differences in behavioral and cardiovascular reactivity to emotive stimuli and their relationship to cognitive flexibility in a primate model of trait anxiety. Front Behav Neurosci 8: 137.

Shively CA, Willard SL (2012). Behavioral and neurobiological characteristics of social stress versus depression in nonhuman primates. Exp Neurol 233: 87-94.

Spinelli S, Chefer S, Suomi SJ, Higley JD, Barr CS, Stein E (2009). Early-life stress induces long-term morphologic changes in primate brain. Arch Gen Psychiatry 66: 658-665.

Suomi SJ (1997). Early determinants of behaviour: evidence from primate studies. Br Med Bull 53: 170-184.

Sylvester CM, Corbetta M, Raichle ME, Rodebaugh TL, Schlaggar BL, Sheline YI et al (2012). Functional network dysfunction in anxiety and anxiety disorders. Trends Neurosci 35: 527-535.

Yokoyama C, Kawasaki A, Hayashi T, Onoe H (2013). Linkage between the midline cortical serotonergic system and social behavior traits: positron emission tomography studies of common marmosets. Cereb Cortex 23: 2136-2145.

Zeredo J, Shiba Y, Roberts AC, Clarke HF (2014). Normalising high trait anxiety in monkeys; the role of hippocampal-medial prefrontal circuitry. 2014 Neuroscience Meeting Planner Program No. 563.15. Online.

Supplementary Information accompanies the paper on the Neuropsychopharmacology website (http://www.nature.com/npp) 